\title{
Topographic Analysis of the Glenoid and Proximal Medial Tibial Articular Surfaces - A Search for the Ideal Match for Glenoid Resurfacing
}

\author{
Anil K. Gupta, MD, MBA*, Bryan Forsythe, MD*, Andrew S. Lee, ${ }^{\star}{ }^{\star}, \dagger$, Joshua D. Harris, \\ MD ${ }^{\star}$, Frank McCormick, MD*, Geoffrey D. Abrams, MD, Nikhil N. Verma, MD ${ }^{\star}$, Anthony A. \\ Romeo, MD. ${ }^{*}$, Nozomu Inoue, MD, PhD ${ }^{\star}, \dagger$, and Brian J. Cole, MD, MBA ${ }^{\star}, \neq$ \\ *Division of Sports Medicine, Department of Orthopaedic Surgery, Rush University Medical \\ Center, Midwest Orthopaedics at Rush, Chicago, Illinois \\ †Orthopaedic Biomechanics Laboratory, Rush University Medical Center, Chicago, Illinois
}

\begin{abstract}
Background-Current knowledge of the appropriate site of osteochondral allograft harvest to match glenoid morphology for the purposes of glenoid resurfacing is lacking. This has led to difficulty with adequately restoring the geometry of the glenoid using current available techniques. The purpose of this study was to quantify the articular surface topography of the glenoid and medial tibial plateau via 3-dimensional (3D) modeling to determine if the medial tibial articular surface provides an anatomic topographic match to the articular surface of the glenoid.
\end{abstract}

Hypothesis-We hypothesized that the medial tibial plateau will provide a suitable osteochondral harvest site due to its concavity and anatomic similarity to the glenoid.

Study Design—Descriptive Laboratory Study

Materials and Methods-Computed tomography (CT) was performed on four cadaveric proximal tibias and four scapulae, allowing for 16 glenoid-tibial comparative combinations. 3D CT models were created and exported into point cloud models. A local coordinate map of the glenoid and medial tibial plateau articular surfaces was created. Two zones of the medial tibial articular surface (anterior and posterior) were quantified. The glenoid articular surface was defined as a best-fit circle of the glenoid articular surface maintaining a two millimeter bony rim. This surface was virtually placed on a point on the tibial articular surface in 3D space. The tibial surface was segmented and its 3D surface orientation was determined with respect to its surface. 3D orientation of the glenoid surface was reoriented so that the direction of the glenoid surface matched that of the tibial surface. The least distances between the point-clouds on the glenoid and tibial surfaces were calculated. The glenoid surface was rotated 360 degrees in one-degree increments and the mean least distance was determined at each rotating angle.

Results-When the centroid of the glenoid surface was placed on the medial tibial articular surface, it covered approximately two-thirds of the anterior or posterior tibial surfaces. Overall,

${ }^{\ddagger}$ Address correspondence to Brian J. Cole, MD, MBA, Department of Orthopaedic Surgery, Cartilage Restoration Center at Rush, Rush University Medical Center Midwest Orthopaedics at Rush, 1611 W Harrison Street, Chicago, IL 60612 
the mean least distance difference in articular congruity of all 16 glenoid-medial tibial surface combinations was $0.74 \mathrm{~mm}$ (standard deviation; $\pm 0.13 \mathrm{~mm}$ ). The mean least distance difference of the anterior and posterior two-thirds of the medial tibial articular surface was $0.72 \mathrm{~mm}( \pm 0.13$ $\mathrm{mm})$ and $0.76 \mathrm{~mm}( \pm 0.16 \mathrm{~mm})$, respectively. There was no significant difference between and the anterior and posterior two-thirds of the tibia with regard to topographic match of the glenoid $(\mathrm{p}=0.187)$.

Conclusion-We describe a novel methodology to quantify the topography of the tibial and glenoid articular surfaces. The findings suggest that the medial tibial articular surface provides an appropriate anatomic match to the glenoid articular surface. Both the anterior and posterior twothirds of the medial tibial articular surface can serve as potential sites for osteochondral graft harvest. This methodology can be applied to future studies evaluating the ideal sites of graft harvest to treat zonal glenoid bone wear and/or loss.

Clinical Relevance-This study provides evidence of a near anatomic topographic match between the medial tibial plateau and glenoid articular surfaces, which has direct clinical application for future biologic osteochondral glenoid resurfacing. This concept has not been described in the literature to date.

\section{Key Terms}

Glenoid; topography; glenoid resurfacing; articular surfaces

\section{Introduction}

The most widely accepted treatment for end-stage primary glenohumeral arthritis is total shoulder arthroplasty (TSA) due to its ability to provide predictable pain relief and improved function. This is particularly the case in elderly and more sedentary patients. TSA for glenohumeral arthritis in young active patients with higher functional demands, however, presents a treatment dilemma. Concerns for component loosening necessitating revision at an early age, high patient expectations, and bone loss and/or soft tissue contracture from prior surgery can all make decision-making for this patient population difficult. ${ }^{1,7}$

Proposed treatment alternatives to TSA for such patients include arthroscopic capsular release, partial or complete synovectomy, microfracture, and non-biological resurfacing hemiarthroplasty. In an attempt to preserve glenoid bone stock, biological soft tissue resurfacing in the form of Achilles allograft, lateral meniscus allograft, and tissue matrices have been introduced. Limited efficacy due to the failure to restore normal anatomy and provide adequate pain relief, however, has led to unsatisfactory outcomes. $4,6,9,13,15,18,20,22$ This is of particular difficulty in situations where there is glenoid bone loss. In an attempt to restore glenoid bone stock, osteochondral allografting of the glenoid has been introduced as an alternative option. ${ }^{10,12}$ Its effective use in shoulder instability and revision arthroplasty has been described. ${ }^{16,17,23}$ Due to a current lack of available glenoid osteochondral allografts, knowledge of sites that provide an anatomic match to the glenoid articular concavity can help advance this technique when used for the purposes of articular-sided resurfacing. 
To the authors' knowledge, there is no data demonstrating an anatomic site that provides an articular surface match to the glenoid for the purposes of total articular osteochondral allograft resurfacing. We hypothesized that the medial tibial plateau will provide an ideal site for glenoid osteochondral allograft resurfacing due to its concave shape and anatomic similarity to the glenoid articular surface. The purpose of this study was three-fold: 1) To quantify the articular surface topography of the glenoid and medial tibial plateau. 2) To reconstruct 3-dimensional models to determine if the medial tibial articular surface provides a topographic match to the articular surface of the glenoid. 3) To compare the anterior and posterior aspects of the medial tibial articular surface to determine if one zone has a more congruent topographic match.

\section{Materials and Methods}

Institutional Review Board exemption was obtained as this is a basic science biomechanical computational analysis using cadaveric specimens.

\section{Specimen Preparation}

Fresh frozen human cadaveric proximal tibias and scapulae without evidence of osteoarthritis were used for this study (four proximal tibias and four scapulae). No specimens were from the same cadaver. Computed Tomography (CT) (Volume Zoom, Siemens, Malvern, PA) images were acquired in the coronal and sagittal planes using $0.625 \mathrm{~mm}$ contiguous slices $(120 \mathrm{kV}, 100 \mathrm{~mA}, 1.0 \mathrm{sec}$ duration, $20 \mathrm{~cm}$ field of view, $512 \times 512$ matrices). Three-dimensional (3D) CT models of the scapulae and proximal tibias were then created and exported into point-cloud models using a 3D reconstruction software program (Mimics, Materialise Inc., Leuven, Belgium) (Figures 1 and 2). Glenoid pointcloud data sets were created by manual segmentation of the glenoid fossa and cortical rim. Similarly, medial plateau point-cloud data sets were created by manual segmentation of the medial plateau and its cortical rim.

\section{Creation of 3D CT Computer Model of the Glenoid Articular Surface}

In order to define orientation and position of the glenoid articular surface in a quantitative manner, a local coordinate was set on the glenoid. Eigenvectors of the glenoid point-cloud data set were calculated to determine orientation of orthogonal principal axes (X, Y and Zaxes) of the glenoid (Figure 3a). A centroid of the glenoid was also calculated to define the origin of the local coordinate system. An orthogonal coordinate system was then determined for each glenoid model by setting the origin of the coordinate at the centroid. ${ }^{23}$ The most inferior, anterior and posterior points of the glenoid model were determined in the local coordinate system. A circumscribed circle was created based on these three points and the circumcenter was calculated. A new orthogonal local coordinate system was created using the same orientation of the glenoid model with a new origin set at the circumcenter (Figure $3 b)$. The orthogonal coordinates for each point consisting of the glenoid model were then converted to a spherical coordinate system. The glenoid articular surface model was created from the glenoid model by selecting points within a distance of circumscribed circle's radius minus $2 \mathrm{~mm}$ from the origin of the spherical coordinate system (Figure 3c). This was performed to mimic the maintenance of a $2 \mathrm{~mm}$ cortical rim for the purpose of articular 
surface press-fit osteochondral grafting. The cortical rim was then excluded and only the glenoid articular surface points were selected.

\section{Creation of 3D CT Computer Model of the Medial Tibial Plateau Articular Surface}

Similar to the creation of the glenoid articular surface model, a local coordinate system of the medial tibial plateau articular surface was created to define its orientation. Eigenvectors and a centroid were calculated and a local orthogonal coordinate system was determined for each model by setting the origin of the coordinate at the centroid. Coordinates for each point of the medial tibial plateau articular surface were then converted to a spherical coordinate system. The outer margin of the medial plateau was defined by points that had the longest distance from the origin within a virtual cone with a vertex angle of $20^{\circ} .{ }^{8,19}$ The margin of the central zone was defined such that its shape was concentric to the outer margin. The distance between the central margin and the outer margin was larger than the radius of the glenoid articular surface model to be analyzed. The peripheral zone was defined by the area outside of the central zone. The central zone was further divided into two zones defined by an angular parameter in the spherical coordinate of each point (posterior zone: $-90^{\circ}$ to $+90^{\circ}$, and anterior zone: $+90^{\circ}$ to $+270^{\circ}$ ) (Figure 4 ).

\section{Glenoid-Medial Tibial Plateau 3D Articular Surface Geometry Matching}

Three-dimensional surface geometry was compared between the glenoid and medial tibial plateau articular surface models for 16 glenoid-tibial comparative combinations (4 glenoid models $\times 4$ tibial models). The glenoid articular surface model was virtually placed on the surface of the medial tibial plateau model so that the centroid of the glenoid merged at a point in the central zone of the tibial plateau. Orientation of the glenoid articular surface model was adjusted so that an axis perpendicular to it matched that of the tibial surface. The "footprint" underneath the glenoid articular surface model was defined as a circle with the radius of the glenoid. This was then placed on the tibial articular surface model (Figure 5a). Orientation of the "footprint" was determined by a vector oriented perpendicular to it. The glenoid articular surface model was then reoriented so that the normal vectors of the glenoid articular surface model and the "footprint" coincided (Figure 5b).

Distances between one point on the glenoid articular surface model and all points on the "footprint" surface were calculated in 3D space. The "least-distance" was defined as the shortest distance from the point in question to the footprint. This procedure was repeated for all points in the glenoid articular surface model. A mean value of the least-distances was calculated for each position and each orientation of the glenoid articular surface model. ${ }^{8,19}$ A perfect congruent match would yield a "least-distance" of $0.0 \mathrm{~mm}$ for the given data points on the simulated articular surfaces.

The glenoid articular surface model was then rotated 360 degrees around the axis perpendicular to the articular surface in one degree increments and the least-distance was calculated at each rotating angle (Figure 5c). The position of the glenoid articular surface model was then moved throughout the entire medial tibial plateau articular surface model and the least-distance was calculated at each position (Figure 6). This was performed for both the anterior and posterior zone of the medial tibial plateau by using the centroid of the 
simulated graft and moving it throughout the anterior two-thirds and posterior two-thirds of the tibia (within the margins of the tibial cortical rim). For each position that the simulated graft was moved, the articular surfaces were compared for match with respect to all data points. The mean and standard deviation of the least-distances for the articular surfaces was calculated for each of the 16 glenoid-tibial model combinations. The mean and standard deviation of the least-distances were also calculated to compare the anterior and posterior zones with respect to "goodness of fit". A perfect match would be a least-distance of zero, while a mean least-distance of less than one millimeter would be considered a near-anatomic match.

\section{Statistical Analysis}

A non-parametric Wilcoxon signed rank test was performed to compare the findings between the anterior and posterior aspects of the medial tibial articular surface with respect to the glenoid. A P-value of less than 0.05 was considered statistically significant.

\section{Results}

The radii of the glenoid articular surface models were $11.0 \mathrm{~mm}, 12.0 \mathrm{~mm}, 12.3 \mathrm{~mm}$ and 13.5 $\mathrm{mm}(12.2 \pm 1.0 \mathrm{~mm}$; mean $\pm \mathrm{SD})$. Anterior-posterior maximum distances of the medial tibial articular surfaces were $39.2 \mathrm{~mm}, 39.7 \mathrm{~mm}, 48.2 \mathrm{~mm}$ and $54.2 \mathrm{~mm}(45.4 \pm 7.4 \mathrm{~mm})$. The most anterior or posterior positions of the centroids of the glenoid model were $27.4 \% \pm$ $4.4 \%$ of the antero-posterior diameter of the medial tibial plateau.

Overall, the mean least-distances between the glenoid and medial tibial plateau surfaces of all 16 glenoid-medial tibial surface combinations was $0.74 \pm 0.13 \mathrm{~mm}$. When the centroids of the glenoid model were located at the anterior and posterior zones, the mean leastdistances of the anterior and posterior zones of the medial tibial articular surface were $0.72 \pm$ $0.13 \mathrm{~mm}$ and $0.76 \pm 0.16 \mathrm{~mm}$, respectively. These results indicate a less than $1 \mathrm{~mm}$ difference in the topographic articular surface match between the medial tibial plateau and the glenoid, where the ideal match would be a mean difference of $0.0 \mathrm{~mm}$. There was no significant difference between and the anterior and posterior two-thirds of the tibia with regard to the topographic match of the glenoid $(\mathrm{p}=0.187)$ (Table 1 and Appendix A).

\section{Discussion}

Topographic analysis, or the study of surface shapes, is a planetary geographic scientific methodology that has been utilized for greater than 400 years and plays an integral role in each country's cartography worldwide. The topographic anatomy of the human body continues to be elucidated. Specific topographic analysis of the human skeletal system is a relatively new concept. Knowledge of the human bony topography in three-dimensional orientation is crucial for orthopaedic surgeons to plan successful operative interventions.

An ideal articular surface match for the purposes of resurfacing would be one in which there is a perfect congruous relationship between all points of the two surfaces in question. This is true of procedures involving osteochondral grafting of the distal femoral articular surface in 
the knee. Failure to congruously match the graft to the patient's native anatomy can lead to eccentric loading, altered contact pressures, and early failure of the graft. ${ }^{3}$

The purpose of this study was to utilize a novel topographic methodology to quantify, compare and contrast the topographic match between the medial tibial plateau and glenoid articular surfaces. Based on the comparison of 16 proximal tibial-glenoid combinations, the medial tibial articular surface provides a near anatomic match to the glenoid articular surface (Figure 7). Both the anterior and posterior two-thirds of the medial tibial articular surface can serve as potential sites for osteochondral graft harvest as the data demonstrates a similar match between the anterior and posterior two-thirds of the medial tibial articular surfaces with that of the glenoid and no significant difference between the two.

Osteochondral allograft resurfacing of the glenoid is a concept that remains in its infancy. There have been several series and reports utilizing iliac crest, femoral head, and distal tibial allografts in the setting of recurrent instability with associated anterior-inferior bone loss. ${ }^{5,11,16,21}$ There is limited data, however, on glenoid osteochondral allograft resurfacing in the setting of significant osteochondral wear or large defects of the articular surface. This is largely in part due to the lack of understanding of the ideal match for glenoid articular surface anatomy. Such lesions can be idiopathic or result from prior trauma, surgery (including prominent anchors, intra-articular pain pumps, thermal chondrolysis), infection, or primary and inflammatory arthritides. ${ }^{6,7,9,14}$ When such lesions present in young, active patients with demanding lifestyles, hemiarthroplasty and/or total shoulder arthroplasty may not be the best option. Gobezie et al described an all-arthroscopic total shoulder resurfacing technique using cadaveric humeral head and proximal tibial condyle. They reported effective short-term results in 10 patients but long-term results are pending. ${ }^{11}$

Clinically, the ideal match that would allow for long-lasting fixation without failure in the setting of glenoid resurfacing has not been determined. A perfect match with anatomic restoration of the glenoid articular surface would be ideal. Fixation failure given potential anatomic mismatch is a legitimate concern. In a biomechanical in vitro model evaluating human and sheep scapulae, Gerber et al. demonstrated that a press-fit fixation provided adequate primary stability when subjected to repetitive shear stresses. ${ }^{10}$ This concept requires near, if not complete, anatomic matching of the donor and host bed to prevent subsidence or loosening. This study was a sawbone analysis of human scapulae along with sheep scapulae only. Given that our study suggests a near-anatomic match between the medial tibia and glenoid, the next step is to perform an in vitro biomechanical analysis of this concept to determine the minimal necessary match that will survive cyclical loading. In the trauma literature, it has been demonstrated that up to $4 \mathrm{~mm}$ of intra-articular glenoid step-off can be treated non-operatively with good functional outcomes. This is largely inpart due to the wide range of motion that can be substituted for by the freedom of motion allowed by the glenohumeral and scapulothoracic articulations. Biomechanical data assessing mismatch tolerance for the purposes of glenoid resurfacing is not known but can serve as a foundation for future research in this area. ${ }^{2,12}$

A paucity of available glenoid allografts on a large scale as well as increased risk of disease transmission due to axial location has led to the need for alternative osteochondral donor 
sites that can provide a similar anatomic match. A better understanding of host and donor site topographic anatomy as demonstrated in this study plays a crucial role in this understanding. The findings can also be applied to advancing the technique of osteochondral grafting of the glenoid. Strengths of the study include computer-based computational analysis, utilization of a proven effective 3D point cloud creation instrument, ${ }^{8,19,23}$ determination of the centroid of the glenoid articular surface, matching the orientation of the articular surface point clouds, and cross-referencing each tibial cloud with each glenoid cloud to account for any variability that may be present between all combinations. One weakness includes a lack of observer blinding. Additionally, this study could have been strengthened with the use of more specimens as this would allow for more generalizability between the usage of varied glenoids and varied tibias. The availability of cadaveric glenoids with no evidence of arthritis, however, is limited. This was accounted for by comparing all specimen combinations. Lastly, the fact that the specimens utilized had no evidence of osteoarthritis makes the direct correlation between the findings and what surgeons encounter clinically (ie. varied size and depth of osteochondral lesions) more difficult. This, however, was not the purpose of this study. The purpose was to assess the articular congruence of normal tibial articular surface to that of the glenoid. Future studies utilizing this methodology can be performed to evaluate specific sites of the proximal medial tibial articular surface to treat zonal glenoid bone wear and/or loss.

\section{Conclusions}

We describe a novel methodology to quantify the topography of the tibial and glenoid articular surfaces. The findings suggest that the medial tibial articular surface provides an appropriate match to the glenoid articular surface. This knowledge can be directly applied to glenoid resurfacing. Further, the anterior or posterior two-thirds of the medial tibial articular surface serve as potential sites for osteochondral graft harvest. This methodology can serve as a template for future studies evaluating the ideal sites of graft harvest to treat zonespecific glenoid bone wear and/or loss.

\section{References}

1. Adams JE, Sperling JW, Hoskin TL, Melton LJ III, Cofield RH. Shoulder arthroplasty in Olmsted County, Minnesota, 1976-2000: A population-based study. J Shoulder Elbow Surg. 2006; 15:5055. [PubMed: 16414468]

2. Anavian J, Gauger EM, Schroder LK, Wijdicks CA, Cole PA. Surgical and functional outcomes after operative management of complex and displaced intra-articular glenoid fractures. J Bone Joint Surg Am. 2012; 94(7):645-53. [PubMed: 22488621]

3. Bartz RL, Camaric E, Noble PC, Lintner D, Bocell J. Topographic matching of selected donor and recipient sites for osteochondral autografting of the articular surface of the femoral condyles. Am $\mathrm{J}$ Sports Med. 2001; 29(2):207-212. [PubMed: 11292047]

4. Bugbee WD, Convery FR. Osteochondral allograft transplantation. Clin Sports Med. 1999; 18:6775. [PubMed: 10028117]

5. Chapovsky F, Kelly JD IV. Osteochondral allograft transplantation for treatment of glenohumeral instability. Arthroscopy. 2005; 21:1007. [PubMed: 16086562]

6. Cole BJ, Yanke A, Provencher MT. Nonarthroplasty alternatives for the treatment of glenohumeral arthritis. J Shoulder Elbow Surg. 2007; 16:231S-240.

7. Denard PJ, Wirth MA, Orfaly RM. Management of glenohumeral arthritis in the young adult. J Bone Joint Surg Am. 2011; 93(9):885-892. [PubMed: 21543679] 
8. Duan CY, Espinoza Orías AA, Shott S, et al. In vivo measurement of the subchondral bone thickness of lumbar facet joint using magnetic resonance imaging. Osteoarthritis Cartilage. 2011; 19(1):96-102. [PubMed: 21034837]

9. Elhassan B, Ozbaydar M, Diller D, Higgins LD, Warner JJ. Soft-tissue resurfacing of the glenoid in the treatment of glenohumeral arthritis in active patients less than fifty years old. J Bone Joint Surg Am. 91(2):419-424. [PubMed: 19181986]

10. Gerber C, Snedeker JG, Krause AS, Appenzeller A, Farshad M. Osteochondral glenoid allograft for biologic resurfacing of the glenoid: biomechanical comparison of novel design concepts. J Shoulder Elbow Surg. 2011; 20(6):909-916. [PubMed: 21444216]

11. Gobezie R, Lenarz CJ, Wanner JP, Streit JJ. All-arthroscopic biologic total shoulder resurfacing. Arthroscopy. 2011; 27(11):1588-1593. [PubMed: 21958671]

12. Jones CB, Sietsema DL. Analysis of operative versus nonoperative treatment of displaced scapular fractures. Clin Orthop Relat Res. 2011; 469(12):3379-89. [PubMed: 21830167]

13. Longo UG, Berton A, Alexander S, Maffulli N, Wallace AL, Denaro V. Biological resurfacing for early osteoarthritis of the shoulder. Sports Med Arthrosc. 2011; 19(4):380-394. [PubMed: 22089288]

14. McNickle AG, L'Heureux DR, Provencher MT, Romeo AA, Cole BJ. Postsurgical glenohumeral arthritis in young adults. Am J Sports Med. 2009; 37:1784-1791. [PubMed: 19509416]

15. Nicholson GP, Goldstein JL, Romeo AA, et al. Lateral meniscus allograft biologic glenoid arthroplasty in total shoulder arthroplasty for young shoulders with degenerative joint disease. J Shoulder Elbow Surg. 2007; 16:S261-S266. [PubMed: 17507244]

16. Provencher MT, Ghodadra N, LeClere L, Solomon DJ, Romeo AA. Anatomic osteochondral glenoid reconstruction for recurrent glenohumeral instability with glenoid deficiency using a distal tibia allograft. Arthroscopy. 2009; 25(4):446-452. [PubMed: 19341934]

17. Provencher MT, LeClere LE, Ghodadra N, Solomon DJ. Postsurgical glenohumeral anchor arthropathy treated with a fresh distal tibia allograft to the glenoid and a fresh allograft to the humeral head. J Shoulder Elbow Surg. 2010; 19(6):6-11.

18. Richards DP, Burkhart SS. Arthroscopic debridement and capsular release for glenohumeral osteoarthritis. Arthroscopy. 2007; 23:1019-1022. [PubMed: 17868843]

19. Simon P, Espinoza Orías AA, Andersson GB, An HS, Inoue N. In vivo topographic analysis of lumbar facet joint space width distribution in healthy and symptomatic subjects. Spine. 2012; 37(12):1058-64. [PubMed: 22433501]

20. Skendzel JG, Sekiya JK. Arthroscopic glenoid osteochondral allograft reconstruction without subscapularis takedown: technique and literature review. Arthroscopy. 2011; 27:129-135. [PubMed: 21187250]

21. Sugaya H, Moriishi J, Dohi M, Kon Y, Tsuchiya A. Glenoid rim morphology in recurrent anterior glenohumeral instability. Arthroscopy. 2011; 27(1):129-135. [PubMed: 21187250]

22. Van Thiel GS, Sheehan S, Frank RM, et al. Retrospective analysis of arthroscopic management of glenohumeral degenerative disease. Arthroscopy. 2010; 26:1451-1455. [PubMed: 20875718]

23. Watanabe S, Inoue N, Yamaguchi T, et al. Three-dimensional kinematic analysis of the cervical spine after anterior cervical decompression and fusion at an adjacent level: a preliminary report. Eur Spine J. 2012; 21(5):946-955. [PubMed: 22124708] 


\section{What is known about the subject}

No studies have compared these two articular surfaces in regards to topographic match for the purposes of allograft transplantation.

\section{What this study adds to existing knowledge}

This study describes a novel methodology to quantify the topography of the tibial and glenoid articular surfaces. The findings suggest that the medial tibial articular surface provides an appropriate anatomic match to the glenoid articular surface. 


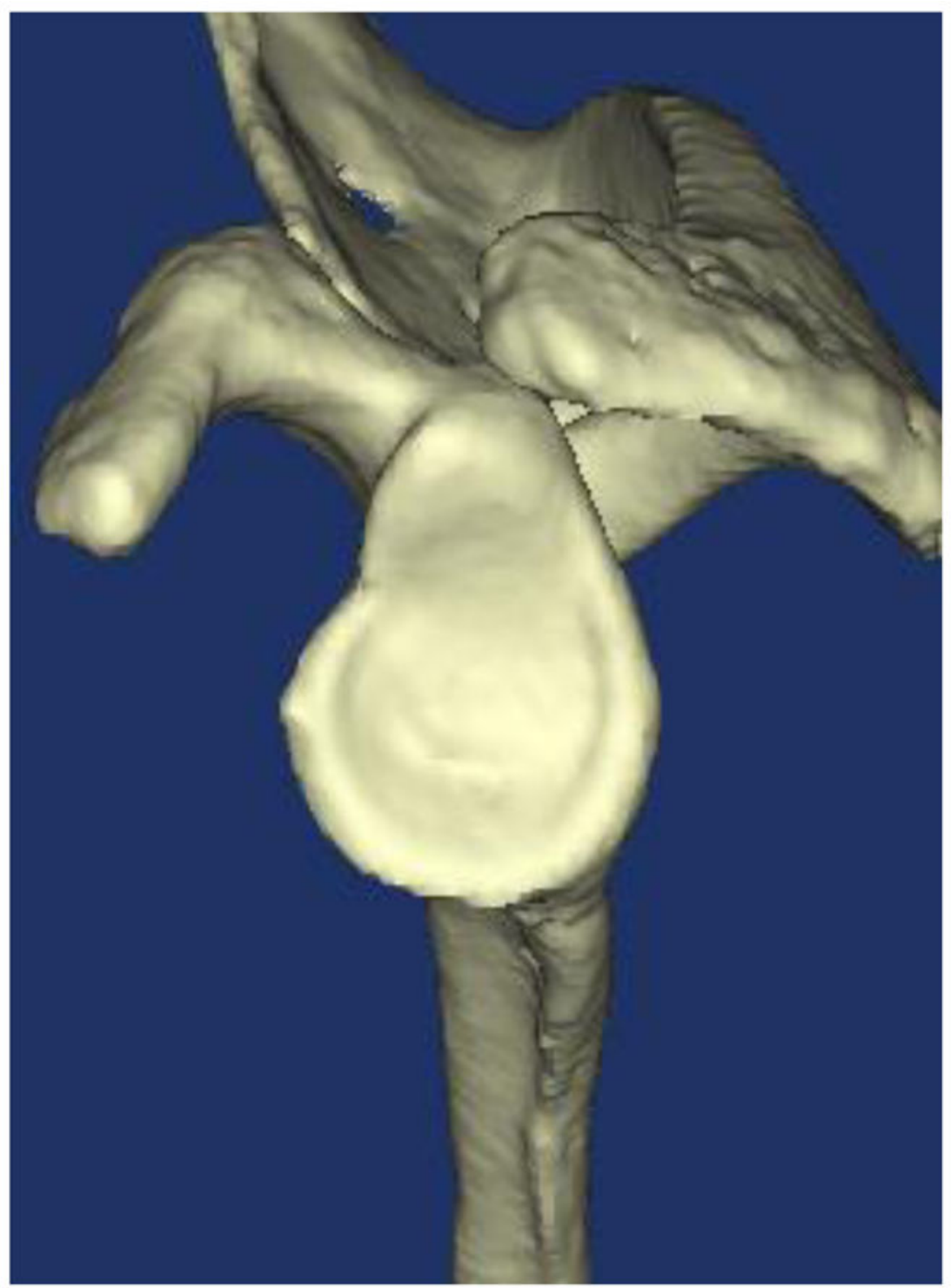

Figure 1.

Three-dimensional (3D) Computed Tomography (CT) model of the scapula. The en face projection of the glenoid surface was utilized for point cloud data analysis. 


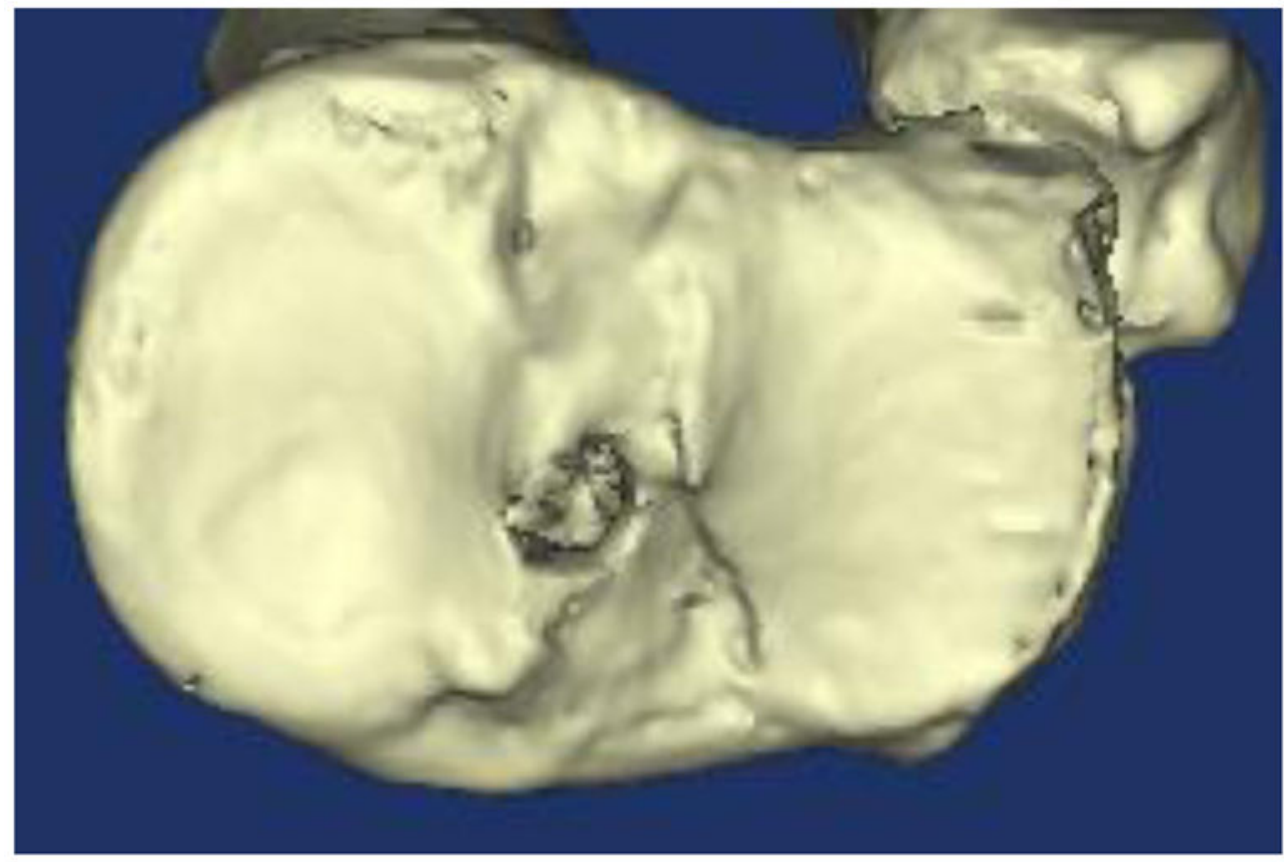

Figure 2.

Three-dimensional (3D) Computed Tomography (CT) model of the proximal tibia. The en face projection of the medial articular surface was utilized for point could data analysis. 


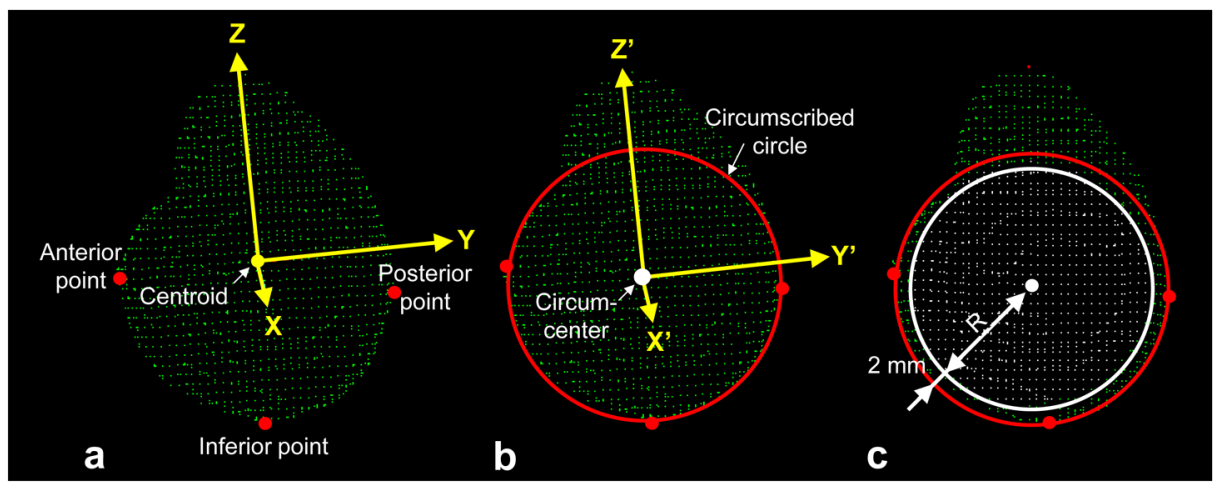

Figure 3.

(A) Point-cloud model of the glenoid surface (green points). An orthogonal local coordinate system (X, Y and Z-axes) of the glenoid was set with the orientation determined by the centroid of the glenoid. The most inferior (along the Z-axis), anterior (along the $\mathrm{Y}$-axis), and posterior (along the Y-axis) points of the glenoid model were determined (red dots). (B) A circumscribed circle (red circle) was created based on these points and the circumcenter (white dot) was calculated. A new orthogonal local coordinate system ( $\mathrm{X}^{\prime}, \mathrm{Y}^{\prime}$ and $\mathrm{Z}^{\prime}$-axes) was set with the same orientation as the $\mathrm{X}, \mathrm{Y}$ and $\mathrm{Z}$-axes and a new origin was determined by the circumcenter. (C) A new circle (white circle) was defined with a center at the circumcenter and a radius (' $R$ ') of the circumscribed circle's radius minus $2 \mathrm{~mm}$ to account for the cortical rim. Points within the new circle were defined as the articular surface points (white points). 


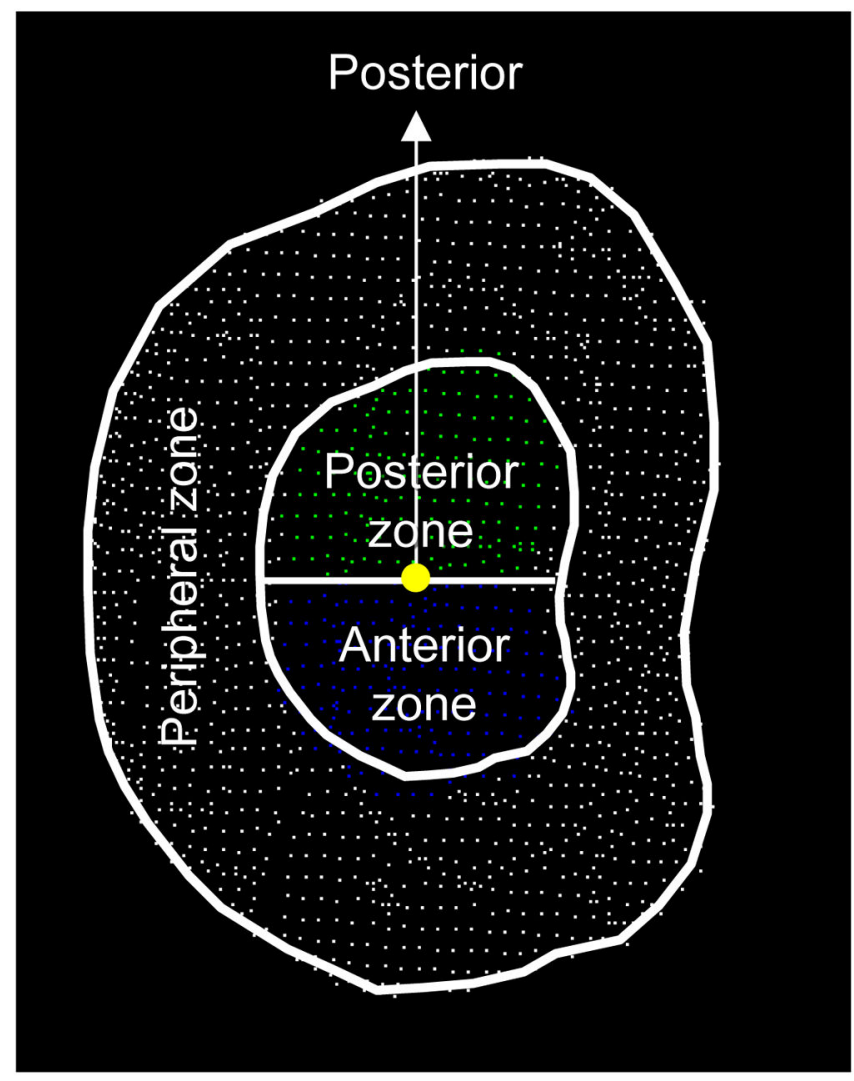

Figure 4.

Medial tibial plateau articular surface point-cloud. The outer margin of the rim was defined. The central zone margin shape was concentric to that of the peripheral zone. The anterior (blue) and posterior (green) zones demonstrate the data points at which the glenoid articular surface model remained within the respective peripheral zone rim during least-distance calculation. 


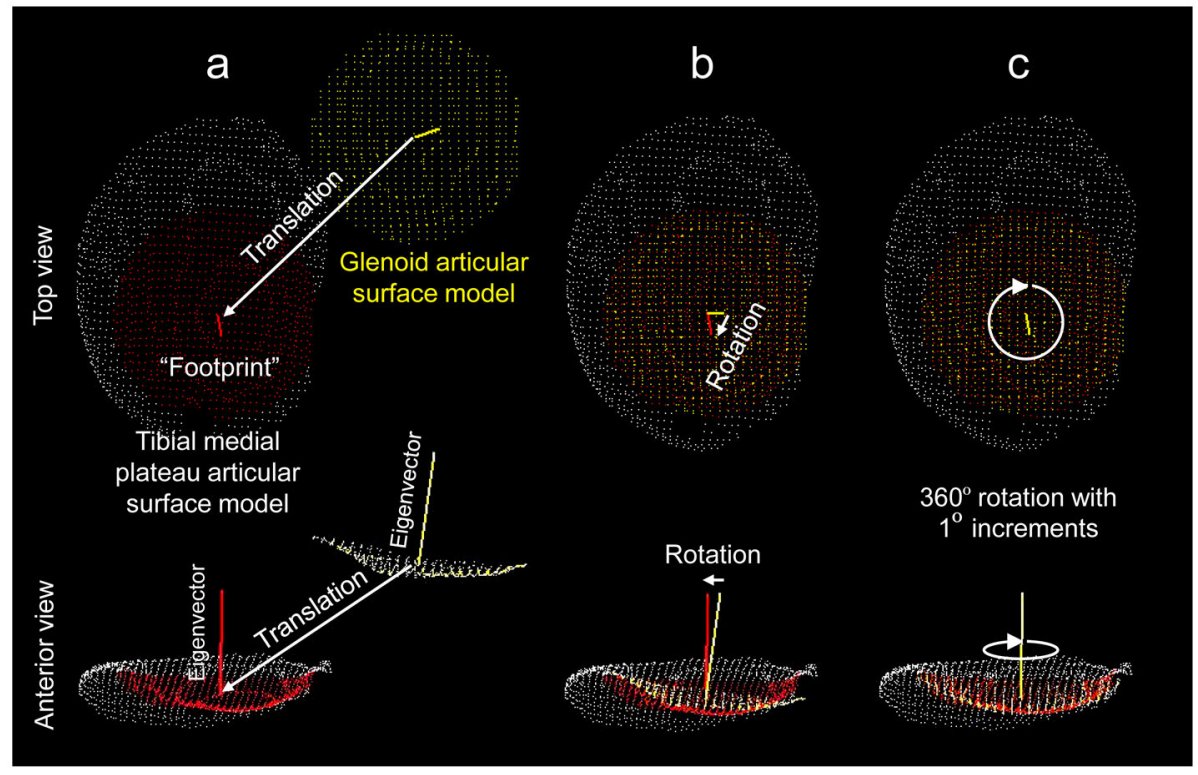

Figure 5.

(A) The "footprint" underneath the glenoid articular surface model (yellow points) was defined as a circle with the radius of the glenoid (red points). This was placed on the tibial articular surface model. The glenoid articular surface model was then superimposed on the "footprint". (B) The eigenvector of the "footprint" and glenoid articular surface model vector were oriented to each other until they matched. (C) The glenoid articular surface model was rotated 360 degrees around the axis perpendicular to the articular surface in onedegree increments and the least-distance was calculated at each rotating angle. 


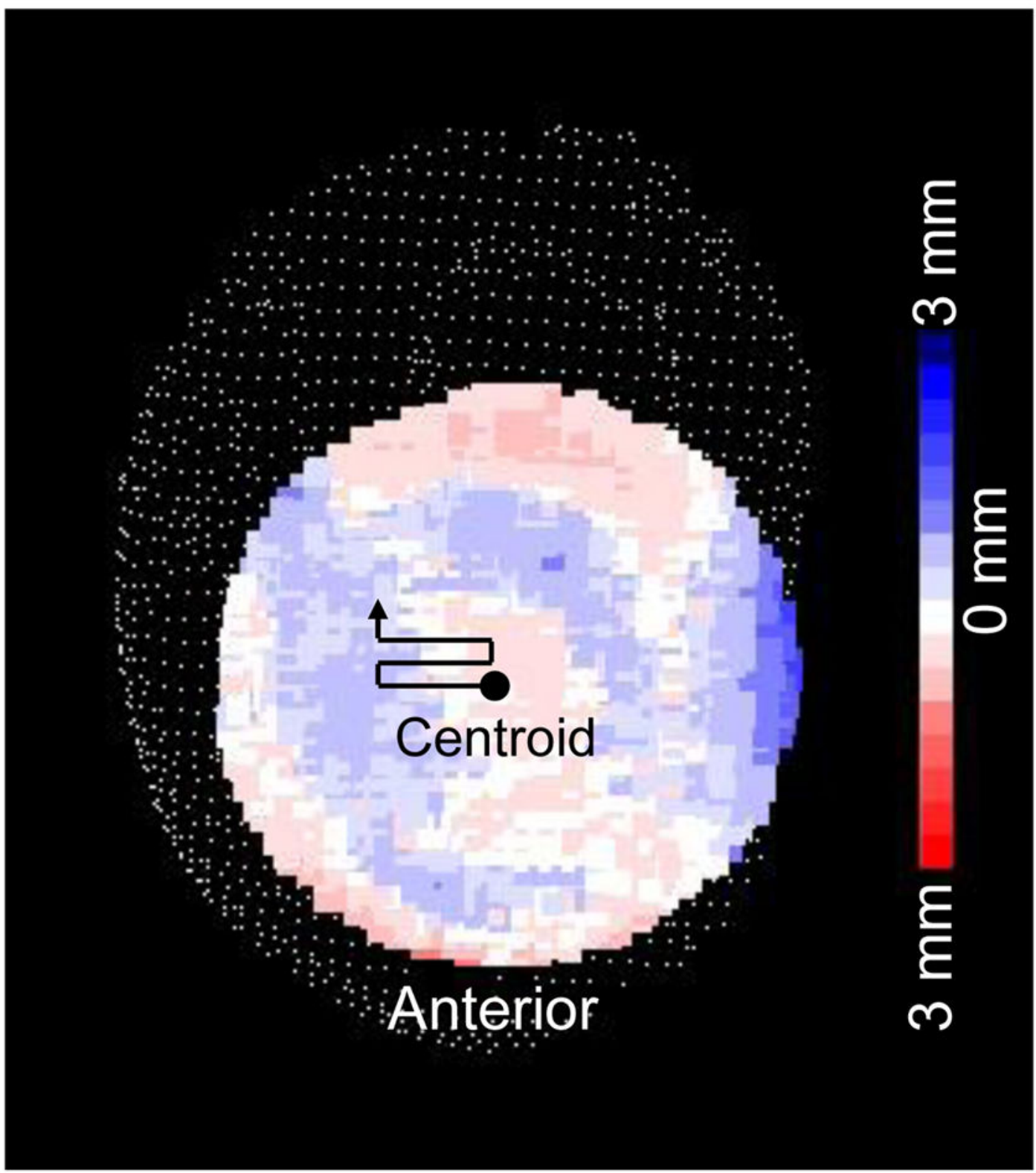

Figure 6.

The glenoid articular surface model was moved throughout the entire medial tibial plateau articular surface model (white points) and the least-distance was calculated at each data point. Distance distribution at one position and the rotational angle is shown. Red gradient color code represents penetration into the tibial joint surface, while blue indicates prominence with respect to the tibial surface. White color code indicates a perfect match between the glenoid and tibial articular surfaces. 


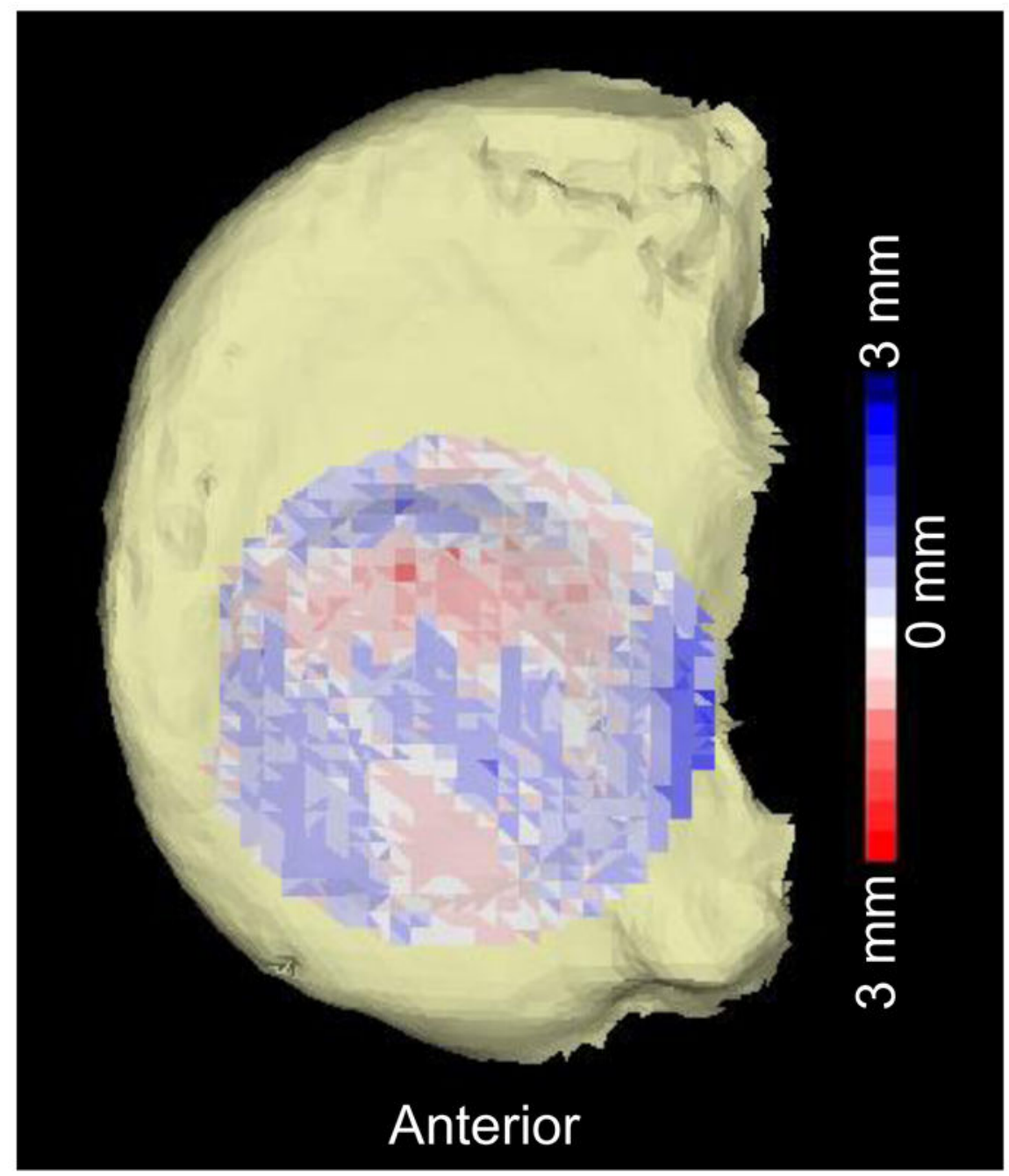

Figure 7.

Three dimensional representation of the distance distribution at "the best match" location and rotational angle of the glenoid articular surface model. This example demonstrates the anterior two-thirds occupancy of the glenoid articular surface model on the tibial surface. Red gradient color code represents penetration into the tibial joint surface, while blue indicates prominence with respect to the tibial surface. White color code indicates a perfect match between the glenoid and tibial articular surfaces. 


\section{Table 1}

Descriptive statistics encompassing all 16 glenoid-tibial combinations. A non-parametric wilcoxon signed rank test was performed to compare the findings between the anterior and posterior two-thirds of the medial tibial articular surface. A P-value of less than 0.05 was considered statistically significant.

\begin{tabular}{|l|l|l|l|}
\hline & Total Articular Surface & Anterior 2/3 & Posterior 2/3 \\
\hline Mean Least Distance & .740 & .720 & .760 \\
\hline Std Dev & .130 & .131 & .160 \\
\hline Min & .567 & .515 & .587 \\
\hline Max & 1.027 & .935 & 1.112 \\
\hline P-value & & & 0.187 \\
\hline
\end{tabular}

